XVI Encontro Sobre a Cultura do Amendoim

15 a 17 de agosto de 2019 na Estação de Eventos Cora Coralina e FCAV/UNESP,

Jaboticabal-SP

\title{
ESTRATÉGIAS DE CONTROLE DA MANCHA-PRETA EM CULTIVARES DE AMENDOIM NA REGIÃO DA ALTA PAULISTA
}

\begin{abstract}
Rodolfo da Silveira Pivaro ${ }^{1}$; Marcos Doniseti Michelotto ${ }^{2}$; Samuel Ferrari ${ }^{3}$; Maycon Ferraz ${ }^{4}$; Gabriel Leonardi Antonio ${ }^{5}$ Ignácio José Godoy ${ }^{6}$
\end{abstract}

\begin{abstract}
${ }^{1}$ Graduando em Agronomia, Bolsista Pibic/Unesp, Dracena, SP, rodolfo_pivaro@ hotmail.com,; ${ }^{2}$ Pesquisador da APTA, Polo Centro Norte, Pindorama, SP, michelotto@apta.sp.gov.br; ${ }^{3}$ Professor da FCAT/UNESP, Dracena, SP, samuel.ferrari@unesp.br; ${ }^{4}$ Graduando em Agronomia, Bolsista Fundag/APTA, Unirp, São José do Rio Preto, SP, maycon.ferraz96@hotmail.com; ${ }^{5}$ Mestrado em Agronomia, bolsista Capes/Unesp, Ilha Solteira, SP, gabriel-leonardi@bol.com.br; ${ }^{6}$ Pesquisador, IAC, Campinas, SP, ijgodoy@iac.sp.gov.br.
\end{abstract}

RESUMO: O objetivo do trabalho foi avaliar diferentes estratégias de aplicação de fungicidas no controle da mancha-preta em cultivares de amendoim na região da Alta Paulista. O experimento foi instalado em área experimental da UNESP, Campus de Dracena, SP. O delineamento experimental foi em esquema fatorial, em blocos casualizados, com 4 repetições. O primeiro fator consistiu de quatro cultivares de amendoim (IAC OL3, IAC OL5, IAC 503 e IAC Top Verde). O segundo fator consistiu de quatro estratégias de aplicação de fungicidas: 1. Testemunha sem controle; 2. Início aos 30 dias após a semeadura (DAS) e reaplicação a cada 10 dias; 3. Início aos 45 dias após a semeadura e reaplicação a cada 15 dias; 4. Monitoramento.A maior incidência da mancha-preta ocorreu na cultivar IAC OL3, intermediária na IAC OL5 e IAC 503 e menor na IAC Top Verde;Para a cultivar IAC OL3, quando realizado a estratégia 30+10DAS observou-se menor mancha-preta e maior produtividade;Para a cultivar IAC 503 a estratégia de aplicação 45+15DAS resultou em bom controle da mancha-preta e maior produtividade;Na cultivar IAC OL5 a melhor estratégia foi o monitoramento ou 45+15DAS;A cultivar IAC Top Verde dispensa o uso de fungicidas.

Palavras-Chave: Arachis hypogaea L.; Produtividade; resistência a doenças.

\section{INTRODUÇÃO}

As doenças fúngicas são provavelmente as de maior importância na cultura do amendoim, Arachis hypogaea L. no mundo (SHOKES \& CULBREATH, 1997). No Brasil, não é diferente, sendo a mancha preta [Cercosporidium personatum (Berk. \& Curtis) Deignton] a doença mais importante no estado de São Paulo, principal produtor do país (MORAES et al., 1997).

As estratégias de manejo recomendadas para essa doença, e que proporcionam melhor controle e maior produção, são aplicações de fungicidas protetores e sistêmicos geralmente em 


\section{Encontro Sobre a Cultura do Amendoim \\ 15 a 17 de agosto de 2019 na Estação de Eventos Cora Coralina e FCAV/UNESP, Jaboticabal-SP}

intervalos de até 15 dias, com início aos 30-45 dias após a semeadura (BARRETO et al., 2007). Com isso, as aplicações de fungicidas podem chagar a dez, ao longo do ciclo de desenvolvimento das plantas de amendoim.

Assim, cultivares resistentes a esta doença torna-se de grande importância para a redução dos custos de produção.

O objetivo do trabalho foi avaliar o efeito das formas de aplicação de fungicidas no controle da mancha-preta em diferentes cultivares de amendoim na região da Alta Paulista.

\section{MATERIAL E MÉTODOS}

O ensaio foi realizado na safra 2018/19 na cidade de Dracena-SP. Foi utilizado o delineamento experimental em blocos casualizados, em esquema fatorial 4 x 4, com quatro repetições. O primeiro fator correspondeu a quatro cultivares (IAC 503, IAC OL3, IAC OL5 e IAC Top Verde) com diferentes características e o segundo fator correspondeu à utilização de diferentes estratégias de controle das doenças (1. Testemunha sem controle; 2. Início das aplicações de fungicida aos 30 dias após a semeadura e reaplicação a cada 10 dias; 3. Início das aplicações de fungicida aos 45 dias após a semeadura e reaplicação a cada 15 dias; 4. Monitoramento: início das aplicações quando do aparecimento das primeiras manchas foliares e reaplicação quando da reinfecção).

As parcelas foram constituídas de quatro linhas de semeadura com $5 \mathrm{~m}$ de comprimento e espaçamento entre linhas de 0,9 m.A semeadura ocorreu no mês novembro, manualmente na densidade de 20 sementes por metro linear.A adubação básica nos sulcos de semeadura foi calculada com base nas características químicas do solo através do resultado da análise química e levando-se em consideração as recomendações da cultura.

Para o controle da mancha preta foi utilizado o fungicida clorotalonil (Bravonil ${ }^{\circledR} 720$ ) na dosagem de $1,5 \mathrm{~L} \mathrm{ha}^{-1}$ do produto comercial e do produto em mistura de piraclostrobina e epoxiconazol $\left(\right.$ Opera $\left.^{\circledR}\right)$ na dosagem de $0,6 \mathrm{~L} \mathrm{ha}^{-1}$ do produto comercial. As pulverizações aéreas dos produtos foram realizadas com pulverizador costal manual com capacidade de $20 \mathrm{~L}$, calibrado para aplicar $300 \mathrm{~L} \mathrm{ha}^{-1}$ de calda.

As avaliações da mancha preta foram realizadas aos 75, 90, 110 e 125 dias após a semeadura (DAS) através de uma escala diagramática com notas de sintomas visuais variando de 1 a 9 de acordo com Surbrahmayan et al. (1980).

Ao final do ciclo foi realizada a colheita das cultivares. Em todas as parcelas foram colhidas as duas linhas centrais e obtendo a produtividade em $\mathrm{kg} \mathrm{ha}^{-1}$.

Os dados foram submetidos à análise de variância pelo teste $\mathrm{F}$, e as médias dos cultivares comparadas pelo teste de Tukey a 5\% de probabilidade. 


\section{Encontro Sobre a Cultura do Amendoim \\ 15 a 17 de agosto de 2019 na Estação de Eventos Cora Coralina e FCAV/UNESP, Jaboticabal-SP}

Tabela 1. Número e data de aplicação de fungicida em função das diferentes estratégias usadas para controle da mancha-preta no experimento. Dracena, SP. Safra 2018/19.

\begin{tabular}{lcc}
\hline $\begin{array}{c}\text { Estratégia de } \\
\text { controle }\end{array}$ & $\begin{array}{c}\mathrm{N}^{\mathbf{o}} \text { de } \\
\text { aplicações }\end{array}$ & $\begin{array}{c}\text { Data das Aplicações } \\
\text { (Dias Após Semeadura) }\end{array}$ \\
\hline Testemunhas & 0 & - \\
45 DAS + 15 & 6 & $24 / 12(45$ DAS); 08/01 (60 DAS); 23/01 (75DAS); 07/02 (90 \\
& $*$ & DAS); 22/02 (105 DAS); 09/03 (120 DAS) \\
Monitoramento & $*$ & DAS); 21/02 (105 DAS); 08/03 (119DAS); 18/03 (129 DAS); \\
& & $09 / 12(30$ DAS); 19/12 (40 DAS); 29/12 (50 DAS); 08/01 (60 \\
30 DAS + 10 & 10 & DAS); 18/01 (70 DAS); 28/01 (80 DAS);07/02 (90 DAS); \\
& & $17 / 02(100$ DAS); 27/02 (110 DAS);09/03 (120 DAS)
\end{tabular}

*IAC Top Verde=1; IAC OL5= 4; IAC 503= 5; IAC OL3= 6 .

\section{RESULTADOS E DISCUSSÕES}

A cultivar IAC Top Verde apresentou as menores notas de sintomas inferiores em relação aos demais cultivares em todos os tratamentos avaliados, evidenciando sua maior resistência à manchapreta (Figura 1). Observou-se também que a cultivar IAC OL3 se mostrou a mais suscetível à doença, por apresentar as notas de sintomas mais elevadas, enquanto as cultivares IAC OL5 e IAC 503 tiveram resultados intermediários.

Para as estratégias de aplicação dos fungicidas, Monitoramento, 45+15DAS e 30+10DAS observou-se uma tendência entre as notas de sintomas da doença entre os cultivares IAC OL3, IAC OL5 e IAC 503. Os mesmos tiveram uma redução nos sintomas apresentados a partir do inicio das aplicações de fungicidas, obtendo notas de sintomas próximas a da cultivar IAC Top Verde (Figura 2). Observou-se também que, com as aplicações aos 30+10DAS as notas de sintomas foram as menores entre os tratamentos. Apesar disso, as aplicações com base no monitoramento se mostraram promissoras devido ocorrer uma diminuição dos sintomas da doença e haver uma redução nas aplicações de fungicida (Figura 1).

Com relação à produtividade, observou-se que a cultivar IAC OL3, quando submetida às aplicações de fungicida no tratamento 30+10DAS, a produtividade de $4.679,9 \mathrm{~kg} \mathrm{ha}^{-1}$ foi significativamente superior aos outros tratamentos. Para a cultivar IAC 503 a estratégia de aplicação $45+15$ DAS resultou na maior produtividade $\left(5.151,9 \mathrm{~kg} \mathrm{ha}^{-1}\right)$ significativamente maior que os demais tratamentos. Os tratamentos 30+10DAS e Monitoramento resultaram em produtividades intermediárias, 4.110,8 $\mathrm{kg} \mathrm{ha}^{-1} \mathrm{e} 3.990,3 \mathrm{~kg} \mathrm{ha}^{-1}$, respectivamente (Figura 3).

Já na cultivar IAC OL5, as maiores produtividades foram observadas nos tratamentos 30+10DAS, 45+15DAS e Monitoramento, diferindo significativamente da testemunha sem controle. Dessa forma, através do monitoramento foram realizadas apenas quatro aplicações de fungicidas sendo mais vantajoso. Por fim, na cultivar IAC Top Verde, as aplicações de fungicidas não 


\section{Encontro Sobre a Cultura do Amendoim}

\section{5 a 17 de agosto de 2019 na Estação de Eventos Cora Coralina e FCAV/UNESP,}

Jaboticabal-SP

proporcionaram aumento de produtividade em relação à ausência de controle, sendo portanto desnecessárias as aplicações de fungicida (Figura 3).
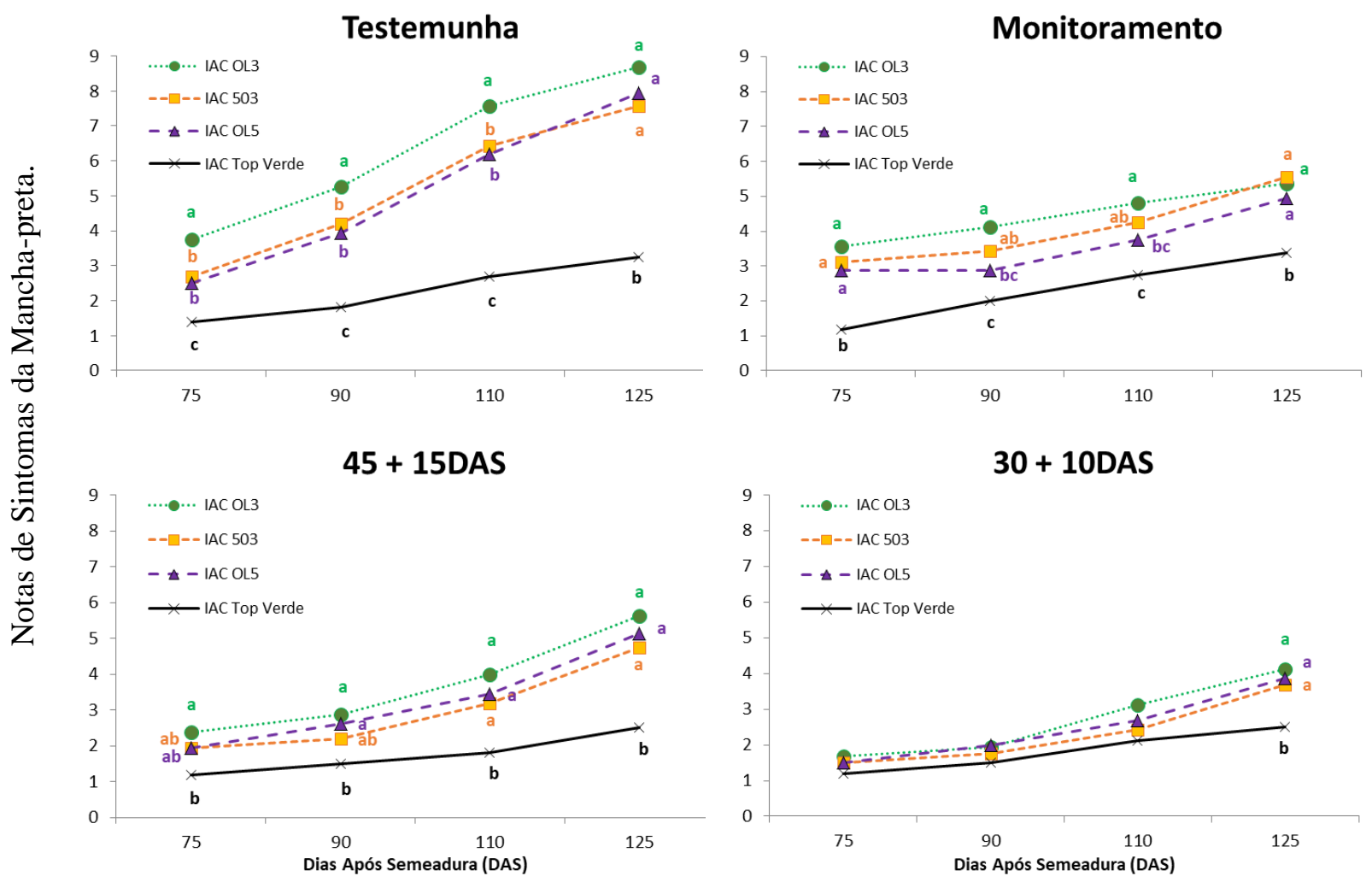

Figura 1. Notas de sintomas de mancha-preta avaliadas aos 75, 90, 110 e 125 dias após a semeadura (DAS) em quatro cultivares de amendoim em função das diferentes estratégias de aplicação dos fungicidas. Dracena, SP. Safra 2018/19. Médias seguidas de letras diferentes em cada data de avaliação diferem entre si pelo teste de Tukey $(p \geq 0,05)$.

\section{IACOL3}

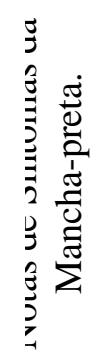

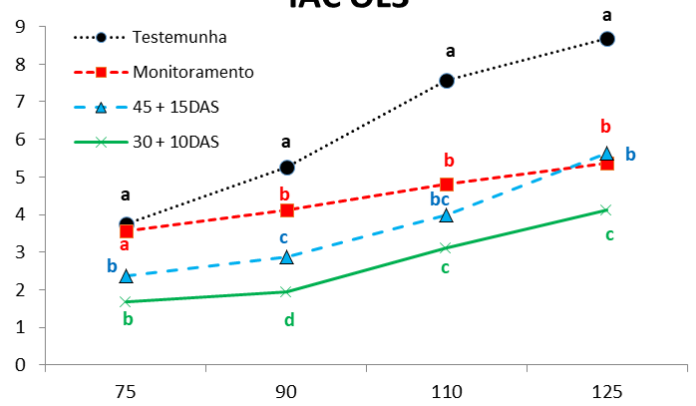

IAC 503

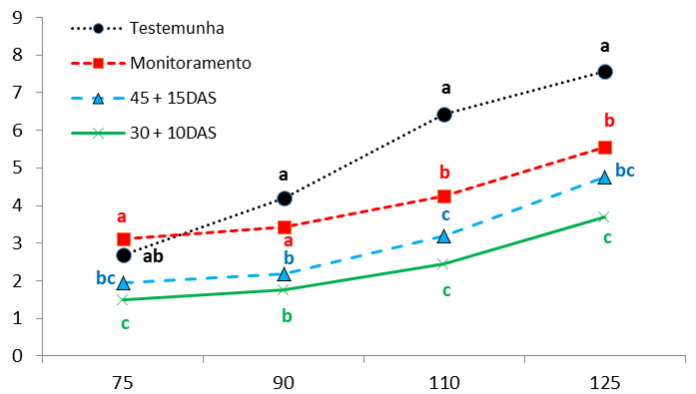




\section{Encontro Sobre a Cultura do Amendoim \\ 15 a 17 de agosto de 2019 na Estação de Eventos Cora Coralina e FCAV/UNESP, Jaboticabal-SP}

IAC OL5

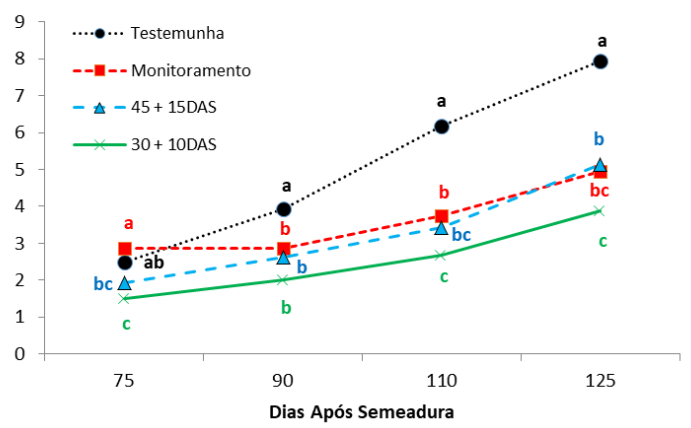

IAC Top Verde

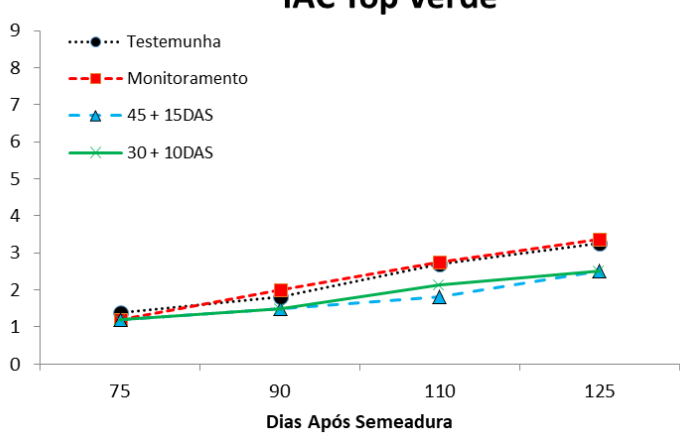

Figura 2. Notas de sintomas de mancha-preta avaliadas aos 75, 90, 110 e 125 dias após a semeadura (DAS) em função das diferentes estratégias de aplicação dos fungicidas dentro de cada cultivar avaliada. Dracena, SP. Safra 2018/19. Médias seguidas de letras diferentes em cada data de avaliação diferem entre si pelo teste de Tukey $(p \geq 0,05)$.
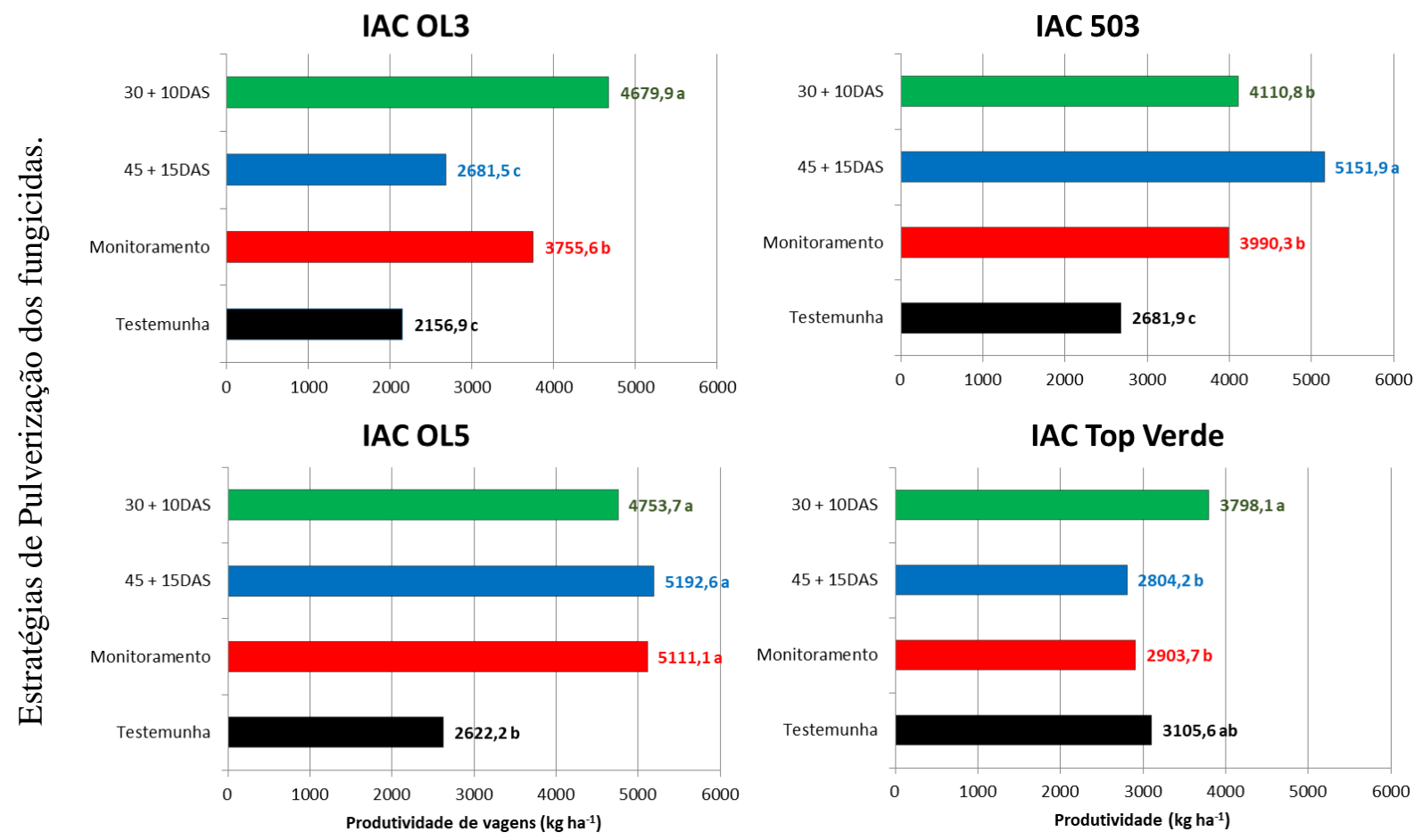

Figura 3.Produtividade de vagens $\left(\mathrm{kg} \mathrm{ha}^{-1}\right)$ em função das diferentes estratégias de aplicação dos fungicidas dentro de cada cultivar avaliada. Dracena, SP. Safra 2018/19. Médias seguidas de letras diferentes em cada cultivar diferem entre si pelo teste de Tukey $(p \geq 0,05)$.

\section{CONCLUSÃO}

A maior incidência da mancha-preta ocorre na cultivar IAC OL3, intermediária na IAC OL5 e IAC 503 e menor na IAC Top Verde;

Para a cultivar IAC OL3, quando realizado a estratégia 30+10DAS observa-se menor manchapreta e maior produtividade;

Para a cultivar IAC 503 a estratégia de aplicação 45+15DAS resulta em bom controle da mancha-preta e maior produtividade; 


\section{Encontro Sobre a Cultura do Amendoim \\ 15 a 17 de agosto de 2019 na Estação de Eventos Cora Coralina e FCAV/UNESP, Jaboticabal-SP}

Na cultivar IAC OL5 a melhor estratégia é o monitoramento ou fungicida a 45+15DAS;

A cultivar IAC Top Verde dispensa o uso de fungicidas.

\section{REFERÊNCIAS BIBLIOGRÁFICAS}

GODOY, I.J. Cultivares de amendoim e diversidades de ambientes. In: XII Encontro sobre a cultura do amendoim, FCAV/UNESP, Jaboticabal-SP, Anais...FCAV: Jaboticabal. p.19, 2015.

MORAES, S. A.; GODOY, I. J. Amendoim - Controle de Doenças. In: ZAMBOLIM, L.; VALE, F. X. R. (eds.) Controle de doenças de plantas: Grandes culturas. Viçosa, Universidade Federal de Viçosa; Brasília, Ministério da Agricultura e do Abastecimento, Suprema Gráfica e Editora Ltda, 1997. v.1, p. 1-49.

SHOKES, F.M. AND A.K. CULBREATH. Early and late leaf spots. In N. Kokalis-Burelle, D.M. Porter, R. RodriguezKabana, D.H. Smith, and P. Subrahmanyam (eds.). Compendium of Peanut Diseases, 2nd Ed. APS Press, St. Paul, MN, pp. 17-20, 1997.

SURBRAHMAYAN P, MEHAAN VK, NEVILL DJ, MACDONALD D. Research on fungal disease of groundnut ICRISAT. In: Proceedings of an International Workshop on Groundnut ICRISAT, Patancheru, AP, India, 1980; pp.193-198. 